\title{
Radiopharmaceutical Therapy of Patients with Metastasized Melanoma with the Melanin-Binding Benzamide ${ }^{131}$ I-BA52
}

\author{
Walter Mier*1, Clemens Kratochwil*1, Jessica C. Hassel ${ }^{2}$, Frederik L. Giesel ${ }^{1}$, Barbro Beijer ${ }^{1}$, John W. Babich ${ }^{3}$, \\ Matthias Friebe ${ }^{4}$, Michael Eisenhut ${ }^{5}$, Alexander Enk ${ }^{2}$, and Uwe Haberkorn ${ }^{1}$ \\ ${ }^{I}$ Department of Nuclear Medicine, University Hospital Heidelberg, Heidelberg, Germany; ${ }^{2}$ Department of Dermatology and National \\ Center for Tumor Diseases, University Hospital Heidelberg, Heidelberg, Germany; ${ }^{3}$ Molecular Insight Pharmaceuticals, Cambridge, \\ Massachusetts; ${ }^{4}$ Bayer Pharma AG, Research Laboratories, Berlin, Germany; and ${ }^{5}$ Department of Radiopharmaceutical Chemistry, \\ German Cancer Research Center, Heidelberg, Germany
}

The performance of cytotoxic drugs is defined by their selectivity of uptake and action in tumor tissue. Recent clinical responses achieved by treating metastatic malignant melanoma with therapeutic modalities based on gene expression profiling showed that malignant melanoma is amenable to systemic treatment. However, these responses are not persistent, and complementary targeted treatment strategies are required for malignant melanoma. Methods: Here we provide our experience with different labeling procedures for the radioiodination of benzamides and report on initial dosimetry data and the first therapeutic application of ${ }^{131}$ I-BA52, a novel melanin-binding benzamide in patients with metastatic malignant melanoma. Twenty-six adults with histologically documented metastasized malignant melanoma received a single dose of $235 \pm 62 \mathrm{MBq}$ of ${ }^{123}$-BA52 for planar and SPECT/CT imaging. Nine patients were selected for radionuclide therapy and received a median of $4 \mathrm{GBq}$ (minimum, $0.51 \mathrm{GBq}$; maximum, $6.60 \mathrm{GBq}$ ) of the $\beta$-emitting radiopharmaceutical ${ }^{131}$ I-BA52. Results: A trimethyltin precursor-based synthesis demonstrated high radiochemical yields in the large-scale production of radioiodinated benzamides required for clinical application. ${ }^{123}$ I-BA52 showed specific uptake and long-term retention in tumor tissue with low transient uptake in the excretory organs. In tumor tissue, a maximum dose of $12.2 \mathrm{~Gy}$ per GBq of ${ }^{131} \mathrm{I}-\mathrm{BA} 52$ was calculated. The highest estimated dose to a normal organ was found for the lung (mean, 3.1 Gy/GBq). No relevant acute or mid-term toxicity was observed with the doses administered until now. Even though dosimetric calculations reveal that the doses applied in this early phase of clinical application can be significantly increased, we observed antitumor effects with followup imaging, and single patients of the benzamide-positive cohort of patients $(3 / 5$ of the patients receiving a dose $>4.3 \mathrm{GBq})$ demonstrated a surprisingly long survival of more than $2 \mathrm{y}$. Conclusion: These data indicate that systemic radionuclide therapy using ${ }^{131}$ IBA52 as a novel approach for the therapy of malignant melanoma is of considerable potential. Future trials should be done to enhance the precision of dosimetry, validate the maximum tolerable dose, and evaluate the effectiveness of the treatment in a prospective manner.

Received Aug. 16, 2012; revision accepted Jun. 17, 2013.

For correspondence or reprints contact: Uwe Haberkorn, University Hospital Heidelberg, Department of Nuclear Medicine, Im Neuenheimer Feld 400, 69120 Heidelberg, Germany.

E-mail: uwe.haberkorn@med.uni-heidelberg.de

${ }^{*}$ Contributed equally to this work.

Published online Nov. 25, 2013.

COPYRIGHT $\odot 2014$ by the Society of Nuclear Medicine and Molecular Imaging, Inc.
Key Words: malignant melanoma; tumor targeting; melanin; radionuclide therapy; personalized medicine

J Nucl Med 2014; 55:9-14

DOI: 10.2967/jnumed.112.112789

$\mathbf{M}$ men and the sixth most common cancer in women in the United States $(1,2)$. The incidence rates of malignant melanoma are increasing rapidly (3). Despite aggressive efforts, the estimated 5-y survival of only $6 \%$ (4) has not been significantly improved. The frequent multifocality of metastases causes poor outcomes; once metastasized, malignant melanomas are no longer curable by surgical and external radiation therapies (5). Unfortunately even with dacarbazine, which is considered the gold standard for the treatment of metastatic melanoma, response rates of less than $20 \%$, with only a small fraction of persisting responses, are achieved (4). In contrast, a systemic therapy based on a targeted internal radiotherapy (radiopharmaceutical therapy) could be effective because melanoma is often sufficiently radiosensitive (6). One prerequisite for this is the selective targeting of melanoma cells with cytotoxic radiopharmaceuticals. $N$-(2-(diethylamino)ethyl)benzamides have been shown to selectively accumulate in melanotic melanoma (7). For radionuclide therapy of malignant melanoma, the benzamide BA52 (benzo(1,3)dioxolo-5-carboxylic acid (4-(2diethylamino-ethylcarbamoyl)-2-iodo-5-methoxy-phenyl)-amide) can be radiolabeled with ${ }^{131} \mathrm{I}$-iodine, a $\beta$-particle-emitting radionuclide with a physical half-life of $8 \mathrm{~d} .{ }^{123} \mathrm{I}$, the $\gamma$-emitting diagnostic counterpart of ${ }^{131} \mathrm{I}$, with a physical half-life of $13.2 \mathrm{~h}$, can be used to identify patients with positive tumor uptake of this drug. Here, we report on the synthesis, radiolabeling, pharmacokinetics, preliminary dosimetry data, and initial therapeutic observations of ${ }^{123} \mathrm{I}-{ }^{131} \mathrm{I}-\mathrm{BA} 52$, a novel benzamide, in adult patients with metastatic malignant melanoma.

\section{MATERIALS AND METHODS}

\section{Patients}

Twenty-six patients (16 men, 10 women; mean age \pm SD, $58 \pm$ $15 \mathrm{y}$; range, 25-81 y) with metastatic, treatment-resistant malignant melanoma were included into the study. All patients gave their written informed consent for benzamide dosimetry and-if warranted-radionuclide therapy. The offer of surrogate treatment to patients without 
further established therapeutic option is in accordance with both the Declaration of Helsinki and our national regulations. The study was approved by the local ethics committee. Before ${ }^{123}$ I-BA52 dosimetry, there was a therapy-free interval of at least $6 \mathrm{wk}$. Patients were included only if PET/CT with ${ }^{18} \mathrm{~F}-\mathrm{FDG}$ results were positive in melanoma lesions within an interval of $2 \mathrm{wk}$.

\section{Preparation of ${ }^{123}$ I-BA52 and ${ }^{131}$ I-BA52}

The synthesis of the stannylated precursor $\mathbf{4}$ is described in the supplemental materials (available at http://jnm.snmjournals.org). Seven hundred micrograms of 4 in $70 \mu \mathrm{L}$ of ethanol were mixed with an aqueous solution of iodide $(60-70 \mu \mathrm{L}$ of $0.1 \mathrm{M} \mathrm{NaOH}$ containing $1.11 \mathrm{GBq}$ of ${ }^{123} \mathrm{I}$ or $10 \mathrm{GBq}$ of ${ }^{131} \mathrm{I}$ [both GE Healthcare]). Twenty microliters of $4.5 \% \mathrm{H}_{2} \mathrm{O}_{2}$ in glacial acetic acid were added. After $10 \mathrm{~min}$, the reaction mixture was purified by semipreparative highperformance liquid chromatography (HPLC). The isolated product was diluted with water and trapped on an Oasis HLB cartridge (Waters). The cartridge was washed with $10 \mathrm{~mL}$ of water, and the product was eluted with $2 \mathrm{~mL}$ of ethanol and $1 \mathrm{~mL}$ of water and neutralized with phosphate buffer. ${ }^{123}$ I-BA52 (600-750 MBq) or ${ }^{131}$ I-BA52 $(6,000-$ $7,500 \mathrm{MBq}$ ) could be isolated. Shortly before injection, the eluate was diluted with $7 \mathrm{~mL}$ of saline and sterile-filtered. Release specifications required less than $5 \%$ free iodide. The analytic reversed-phase HPLC analyses were performed on an Agilent 1100 HPLC system equipped with a $\gamma$-detector using a Chromolith Performance RP-18 endcapped column $(3 \times 100 \mathrm{~mm})$. A linear gradient under the following conditions was used: gradient $(0 \%-100 \%)$ of acetonitrile $(0.1 \%$ trifluoroacetic acid [TFA]) in water (0.1\% TFA) in $15 \mathrm{~min}$; flow rate, 2 $\mathrm{mL} / \mathrm{min} ; 50^{\circ} \mathrm{C}$; absorbance $\lambda, 214 \mathrm{~nm}$.

\section{Dosimetry with ${ }^{123}$ I-BA52}

Planar (anterior and posterior) whole-body scans were obtained $10 \mathrm{~min}, 4 \mathrm{~h}, 24 \mathrm{~h}$, and $48 \mathrm{~h}$ after intravenous injection of $235 \pm 62$ (range, 178-379) MBq of ${ }^{123}$ I-BA52. Because almost all patients were in bad general clinical condition, for ethical reasons we discontinued dosimetry in patients considered BA52-negative based on the 24-h scan. Finally, 1 patient with nearly no tumor uptake and 10 patients covering a wide spectrum of tumor load were evaluated using this protocol. Imaging was done with a medium-energy collimator (energy peak, 159 $\mathrm{keV}$; window, $15 \%$ ) on a Hawkeye $\gamma$ camera (GE Healthcare). Correlation with ${ }^{18} \mathrm{~F}-\mathrm{FDG}$ PET/CT was conducted to identify metastatic lesions.

The whole-body counting rates of the 10-min-after-injection scans, acquired before voiding, were equal to $100 \%$ of the injected dose. Region-of-interest (ROI) analysis was performed in the conjugate view (geometric mean) to determine the counting rate of the metastases and accumulating organs (liver, spleen, kidneys, lungs, and whole body). Maximum tissue uptake (Umax) of radioactivity was calculated from decay-corrected counting rates as a percentage of the injected activity. The mean residence time was then determined from the biologic halflife calculated from the 4-, 24-, and 48-h scans and after extrapolation from the physical half-life of ${ }^{123} \mathrm{I}(13.2 \mathrm{~h})$ to ${ }^{131} \mathrm{I}$ (193 $\mathrm{h}$ ) for organs and tumor lesions with a Umax of $24 \mathrm{~h}$ or less after injection. Dose estimation was not possible for metastases and organs with a Umax greater than $24 \mathrm{~h}$ after injection because of the short half-life of ${ }^{123} \mathrm{I}$. Organ doses were calculated with OLINDA/EXM (8) using the adult male and female phantom, and tumor dose was calculated using the cyclic sphere model with a lesion diameter taken from the correlating $\mathrm{CT}$. Because of the limitations of planar imaging to discriminate doses in the case of organ overlap, tumor dosimetry was performed only for lesions evaluable without relevant background activity, for example, lymph nodes axillar or inguinal and particular bone lesions.

\section{Dosimetry with ${ }^{131}$ I-BA52}

One melanoma patient who was in outstanding good general condition received an extended dosimetry while being under treatment with ${ }^{131}$ I-BA52. Planar whole-body scans were obtained $10 \mathrm{~min}, 4 \mathrm{~h}$, $24 \mathrm{~h}, 48 \mathrm{~h}, 96 \mathrm{~h}$, and $120 \mathrm{~h}$ after injection of $1,400 \mathrm{MBq}$ of ${ }^{131} \mathrm{I}-\mathrm{BA} 52$, which was also revealed to be the upper limit of activity without saturating the counting-rate linearity of the $\gamma$ camera early after injection. Imaging was done with a high-energy collimator (energy peak, $364 \mathrm{keV}$; window, 15\%) on the same camera (1" crystal Hawkeye $\gamma$ camera [GE Healthcare]). The counting-rate efficiency was ascertained as $9.75 \times 10^{-5} \mathrm{MBq} /$ count or 10,256 counts/MBq and was validated to be linear over the range of measured activities, with standard point sources placed beside the lower legs of the patient, respectively. Attenuation correction was not measured, but the transmission was always assumed to be $11.1 \%\left(20 \mathrm{~g} / \mathrm{cm}^{2}\right.$ thickness; attenuation coefficient, $0.11 \mathrm{~cm}^{2} / \mathrm{g}$ at $364 \mathrm{keV}$ ). ROIs for the liver, lung, colon, and metastases were drawn according to Supplemental Figure $1 \mathrm{~A}$, and the geometric mean of the anterior and posterior images were calculated. Both lung and liver present overlap with other tissue. Therefore, the half-life in the lung was determined with an ROI around the left lobe excluding the heart, and background correction was done with an ROI placed in projection to the lateral thoracic wall. For the liver, an elliptic ROI was placed in the center of the organ, and a same-sized ROI was drawn (carefully placed between lung and bowel) in the left upper abdomen for background subtraction. The counts of the background ROIs were halved to avoid overcorrection. The whole-body remainder could not be determined by a whole-body ROI because the result of this procedure would be dominated by the BA52-positive organs and metastases. Therefore, the half-life in the remainder (interstitial fluid, muscle, fat) was assessed with an ROI over the upper leg. Quantification of eye uptake was done from the ventral position. In each scan and per eye, 2 ROIs were drawn. Because of the limited resolution of planar scintigraphy, spill-out effects are relevant for small objects. Therefore, a widely overlapping 300pixel ROI was centered over each eye, collecting all $\gamma$ emissions with possible origin in the eyeball. This overlap necessarily also includes a large amount of background activity, which was subtracted by the counts of a second 300-pixel ROI circle enclosing the inner ROI (Supplemental Fig. 1B). For attenuation correction, it was assumed that the activity would be in a depth of $1 \mathrm{~cm}$ mean. On the basis of earlier experience in swine animal models (M. Friebe, unpublished data, 2010), we assumed that the whole activity was accumulated only in the pigment of the choroid and the cilliar body, with both structures contributing equally to the total uptake. On the basis of textbook values for human beings, the area of the choroid was assumed as $12.5 \mathrm{~cm}^{2}$ (thickness, $0.02 \mathrm{~mm}$ ); the retina was assumed with a thickness of $0.2 \mathrm{~mm}$, with direct contact to the choroid. With an open pupil, the pigmented epithelia of the cilliar body was assumed to be elliptic, with a widely homogeneous distribution of activity. As illustrated in Supplemental Figure 1C, 1 pulmonal metastasis on the left side had background correction by a contralateral ROI, and 1 metastasis in projection to the right scapula had significant lung overlap, which was corrected by a pulmonal background ROI on the left side. A metastasis in the axillar region presented no relevant organ overlap, and background correction was performed by a mirrored ROI to the contralateral side. The calculated residency times were translated into organ doses using the software OLINDA (8). For the bladder, the 4-h voiding model of the software was used.

\section{Patient Selection and Therapy with ${ }^{131}$ I-BA52}

From the 26 patients scheduled for BA52 uptake screening, 10 patients were determined eligible for radionuclide therapy based on a positive tumor targeting within the preceding ${ }^{123}$ I-BA52 scans. Despite an intense accumulation of ${ }^{123}$ I-BA52, 1 patient could not receive treatment because of a rapid worsening of his general clinical condition.

The therapy with ${ }^{131} \mathrm{I}-\mathrm{BA} 52$ was administered as an intravenous infusion of 3,900 $\pm 2,000$ (range, $510-6,600$ ) $\mathrm{MBq}$ of ${ }^{131} \mathrm{I}-\mathrm{BA} 52$. 
The 364-keV $\gamma$ emission of ${ }^{131}$ I was used to acquire whole-body scans $72-96 \mathrm{~h}$ after the therapeutic injection to validate the positive tumor uptake and retention and relative organ uptake as compared with the 24 - or 48 -h images acquired with ${ }^{123}$ I-BA52. The thyroid uptake was blocked with orally administered sodium perchlorate (Irenat; Bayer Vital GmbH) $1 \mathrm{~h}$ before ${ }^{123} \mathrm{I}-{ }^{131} \mathrm{I}-\mathrm{BA} 52$ injection and during the week after tracer administration $(3 \times 400 \mathrm{mg} / \mathrm{d})$.

\section{RESULTS}

\section{Reliable Synthesis for Clinical Activities of ${ }^{131}$ I-BA52}

The labeling of BA52 was first attempted by direct labeling of its noniodinated precursor. The best results were obtained by reacting the corresponding nonhalogenated benzamide derivative in TFA in the presence of Tl(TFA) $)_{3}$, followed by radioiododethallation. The thallium trifluoroacetate method involves the formation of an intermediate thallium precursor that undergoes nucleophilic substitution with radioiodide. This process allowed the regioselective iodination. Unfortunately, this process was not satisfactory for the large-scale production because of poor reproducibility and low radiochemical yields. Consequently, attempts were made to synthesize a trimethyltin precursor of BA52. Radioiododestannylation of trialkyltin precursors allows the synthesis of carrierfree iodinated compounds by regioselective iodination. The trimethyltin precursor was prepared in excellent yields by reacting the corresponding halogenated benzamide precursor with hexamethyldistannane in the presence of $\mathrm{Pd}\left(\mathrm{PPh}_{3}\right)_{2} \mathrm{Cl}_{2}$. The synthetic pathways to obtain trimethyltin precursor $\mathbf{4}$ are presented in the supplemental material. The stannyl precursor $\mathbf{4}$ was characterized in detail by nuclear magnetic resonance (NMR) spectroscopy (1D ${ }^{1} \mathrm{H}$ and ${ }^{1} \mathrm{H}$-dec.; ${ }^{13} \mathrm{C}$ and DEPT-135; 2D COSY-LR-45; ${ }^{1} \mathrm{H} /{ }^{13} \mathrm{C}$-heteronuclearsingle quantum coherence (HSQC); ${ }^{1} \mathrm{H} /{ }^{13} \mathrm{C}$-heteronuclearmultiple bond correlation (HMBC) [optimized for 6-Hz coupling]). Radioiododestannylation of $\mathbf{4}$ was performed with ${ }^{123} \mathrm{I}$ or ${ }^{131} \mathrm{I}$ for either scintigraphy or therapy, respectively, and the trimethyltin precursor using hydrogen peroxide as the oxidant as shown in Figure 1.

The carrier-free radioiodinated benzamides were purified by HPLC and obtained with a radiochemical yield greater than $50 \%$ after isolation. The coinjections of the radiolabeled material with an independently prepared iodinated standard, which had been characterized by NMR spectroscopy, confirmed the identity of the radioiodinated compounds. The product reconstituted in aqueous solution was unstable. However, the radioiodinated benzamide was found to be stable in ethanol. When reconstituted shortly before administration, the product can be prepared and administered in high purity.

\section{Tumor Targeting and Dosimetry}

In $38 \%$ of the patients (10/26), ${ }^{123}$ I-BA52 accumulated in metastatic melanoma manifestations, with promising intensity for treatment (Figs. 2 and 3). The mean and SD of the administered mass of ${ }^{123} \mathrm{I}-\mathrm{BA} 52$ was $45 \pm 10 \mu \mathrm{g}$ (range, 20-60 $\mu \mathrm{g}$ ). The mean administered activity was $235 \pm 62 \mathrm{MBq}$ (range, 178-379 MBq). There were no adverse or clinically detectable pharmacologic

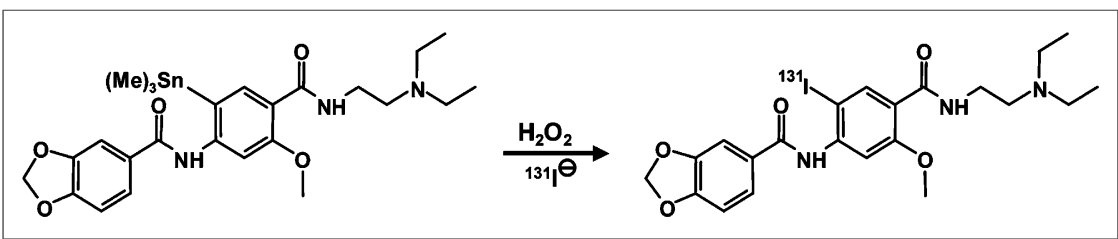

FIGURE 1. Chemical structure of novel melanin-binding benzamide ${ }^{131}$ I-BA52. effects in any of the 26 subjects. Especially, no significant changes in vital signs were observed within $48 \mathrm{~h}$ after administration of the radiopharmaceutical.

The 10-min images were dominated by perfusion (i.e., visualization of vessels and heart). At $4 \mathrm{~h}$, the highest concentration of BA52 was observed in the liver, and the first soft-tissue metastases were visible as well. Because of the slow background clearance, the 4-h-after-injection scans were considered insufficient to determine whether a patient was BA52-positive or not. After $24 \mathrm{~h}$, the perfusion-dependent cardiac uptake had gone. The lung was still visible. The activity inside the liver declined rapidly, obviously due to hepatobiliary excretion because the small intestine became contrasted as early as $4 \mathrm{~h}$ after injection. Once inside the colon, the activity was transported slowly from the ascending colon to the sigmoid, which was still contrasted after $120 \mathrm{~h}$. Melanoma metastases demonstrated long retention of BA52, with increasing tumor-to-background delineation over time especially for brain and lung lesions (Figs. 2 and 3; Supplemental Figs. 1 and 2). However, with a half-life of about $12 \mathrm{~h}$ for ${ }^{123} \mathrm{I}$, the images acquired at $48 \mathrm{~h}$ were degraded by a low counting rate and consecutively more noise. Consequently, the 24-h-after-injection scan was used for treatment stratification.

The ${ }^{123}$ I-BA52 (physical half-life, $13.2 \mathrm{~h}$ )-based dosimetry of 11 patients was sufficient to evaluate the doses to normal organs with early Umax and short biologic half-life such as the liver, lung, kidneys, and spleen. The lungs were the organs with the highest specific doses $(3.2 \pm 2.20 \mathrm{~Gy} / \mathrm{GBq})$, followed by the liver $(1.3 \pm 0.98 \mathrm{~Gy} / \mathrm{GBq})$, the spleen $(0.6 \pm 0.13 \mathrm{~Gy} / \mathrm{GBq})$, and the kidneys $(0.3 \pm 0.11 \mathrm{~Gy} / \mathrm{GBq})$. In extracranial tumor lesions, Umax was measured $24 \mathrm{~h}$ after injection; however, the biologic half-life in melanoma metastases is a multiple of the physical halflife of ${ }^{123}$ I. Therefore, a relevant error magnification should be considered for the extrapolation from ${ }^{123} \mathrm{I}$ to ${ }^{131} \mathrm{I}$. The effective half-life of ${ }^{131}$ I-BA52 in melanoma metastases was determined close to or even exceeding the physical half-life of ${ }^{131} \mathrm{I}$. That means that the biologic washout from tumor lesions was still partially compensated by positive tumor accumulation even beyond $24 \mathrm{~h}$ after injection. Taking into account the 39-h half-life of the whole-body remainder, daily whole-body counting with a $\gamma$ probe, and posttherapeutic scans with ${ }^{131} \mathrm{I}$-BA52, we considered an effective tumor half-life between 3 and $7 \mathrm{~d}$ to be a more realistic and cautious assumption. The estimated doses to the evaluable extracranial metastatic lesions were $5.51 \pm 3.6 \mathrm{~Gy} / \mathrm{GBq}$, with a range from 1.4 to 12.2 , but should be interpreted with care because of the mentioned limitation. Brain metastases demonstrated slower uptake kinetics, with a Umax greater than $48 \mathrm{~h}$ after injection, and not even a rough approximation of tumor dosimetry can be given.

Within the ${ }^{131}$ I-BA52 (physical half-life, 193 h)-based dosimetry of 1 patient (presented in Supplemental Fig. 3), in addition regions with a delayed Umax could be evaluated and the effective half-life of organs and tumor sites was measured directly with the therapeutic compound. Therefore, this case provides the most reliable dosimetry data of our study. The Umax was found with whole-organ ROIs at $4 \mathrm{~h}$ after injection with $10.4 \%$ of the injected dose (\%ID) for the liver and $7.4 \%$ ID in the lung. The liver half-life was $34 \mathrm{~h}$ and the lung halflife $137 \mathrm{~h}$. Consecutively, the organ residency times were determined to be $14.6 \mathrm{~h}$ 


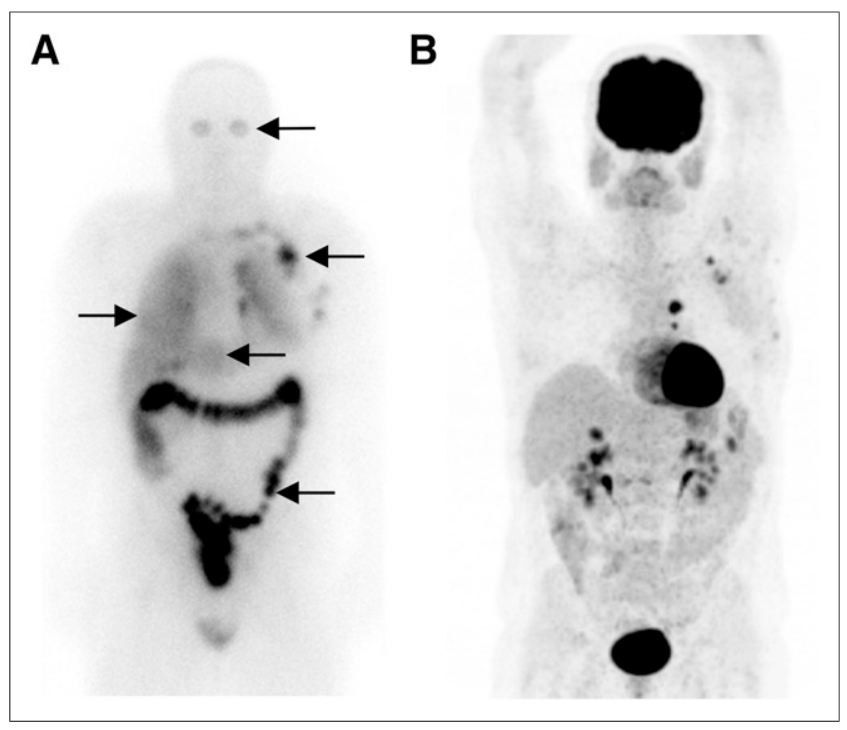

FIGURE 2. (A) Biodistribution of ${ }^{131} \mid$-BA52 in patient with malignant melanoma. Arrows indicate uptake of benzamide in melanin-bearing cells of eyes, specific uptake in tumor lesions (auxiliary) as identified by ${ }^{18} \mathrm{~F}-\mathrm{FDG}$ (B), unspecific uptake in lungs, and elimination through liver and bowel.

for lung and $5.1 \mathrm{~h}$ for liver, translating into doses of $1.8 \mathrm{~Gy} / \mathrm{GBq}$ for the lung and $0.5 \mathrm{~Gy} / \mathrm{GBq}$ for the liver. The hepatic activity demonstrated rapid clearance into the small intestine (Umax $4 \mathrm{~h}$ after injection). The delayed images presented a rather slow transport from the ascending colon ( $24 \mathrm{~h}$ after injection) to the transverse colon (48 $\mathrm{h}$ after injection) and descending colon (96$120 \mathrm{~h}$ after injection). The Umax in the sigmoid was not reached until $120 \mathrm{~h}$ after injection; therefore, only the residency time (4.5 h) for the more proximal colon has been calculated, translating into a colon dose of 1.1-1.4 Gy/GBq. However, the slow transport through the colon implicates that BA52 sticks to the mucosa, and the bowel radiation might be underestimated assuming a homogeneous distribution inside the intestine. The whole-body remainder (interstitial fluid, muscle, fat) was calculated with $39 \mathrm{~h}$. For the eyes, the Umax was found $24 \mathrm{~h}$ after injection with 0.023 $\%$ ID on the right and $0.021 \%$ ID on the left side. The effective

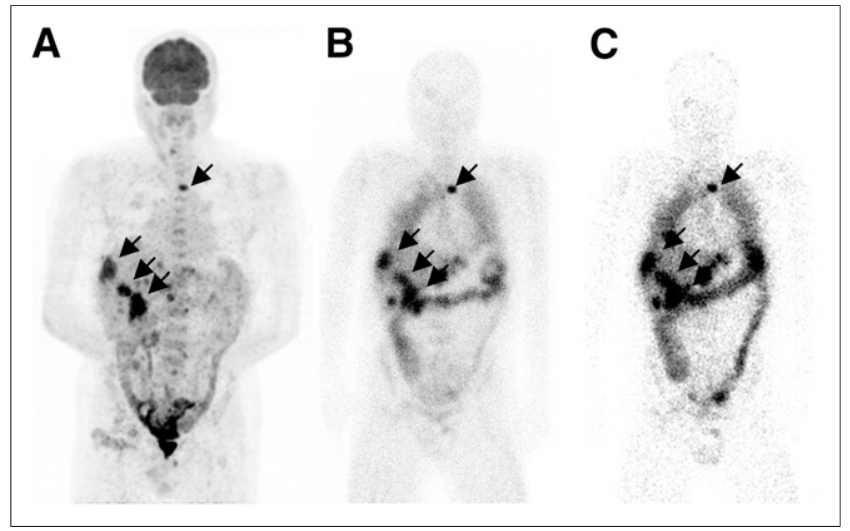

FIGURE 3. (A) A 68-y-old patient with liver and bone (sternum) metastases of malignant melanoma. Tumor lesions were identified by ${ }^{18} \mathrm{~F}-\mathrm{FDG}$ PET. Tumor residence time and elimination pathway were determined with scans of 123 -BA52 at $4 \mathrm{~h}$ (B) to $24 \mathrm{~h}$ (C) after injection. Side-by-side reading with ${ }^{18} \mathrm{~F}-\mathrm{FDG}$ demonstrates highly specific accumulation and also long retention time in tumor tissue. Increasing contrast of bowel corresponds to hepatoenteral excretion. half-life was $151 \mathrm{~h}$ in the right and $169 \mathrm{~h}$ in the left eye. Consecutively, residency times were 0.058 and $0.051 \mathrm{~h}$, respectively. On the basis of the used eye model, the dose per hour residency time would be $300 \mathrm{~Gy} / \mathrm{GBq} / \mathrm{h}$ for the choroid, $100 \mathrm{~Gy} / \mathrm{GBq} / \mathrm{h}$ for the retina, and $210-570 \mathrm{~Gy} / \mathrm{GBq} / \mathrm{h}$ (individual size of cilliar body not known) for the cilliar body. However, the residency time of the whole eye $(0.054 \mathrm{~h})$ was divided for the cilliar body and choroid, resulting in only a 0.027 -h residency time. Two metastases were big enough to neglect underestimation of maximum tumor uptake by partial-volume effects. Together with consistent counting rates over $120 \mathrm{~h}$, the calculated tumor doses of $6.0-9.3 \mathrm{~Gy} / \mathrm{GBq}$ are quite reliable. When entering the given values for the organs with measurable BA52 accumulation into OLINDA and assuming a homogeneous distribution within the remainder tissue, all other organs stay below $0.2 \mathrm{~Gy} / \mathrm{GBq}$, including the bone marrow with $0.1 \mathrm{~Gy} / \mathrm{GBq}$.

However, the $\gamma$ emission from the lungs, liver, and colon would contribute approximately $80 \%$ of the dose to the bone marrow. Under a more conservative assumption that up to $10 \%$ of the remainder might be localized in the red bone marrow, the potential radiation exposure increases to $0.2 \mathrm{~Gy} / \mathrm{GBq}$. The equivalent whole-body dose was calculated as $0.7 \mathrm{~Gy} / \mathrm{GBq}$. The comparison of the ${ }^{123}$ I- and the ${ }^{131}$ I-BA52-based images (Supplemental Fig. 3) of this patient revealed matchable dosimetry results for rounding to 1 position after the decimal point.

The mean normal organ doses as calculated from the eleven ${ }^{123}$ I-BA52 examinations completed with the doses for large intestine and eyes from one ${ }^{131}$ I-BA52 dosimetry and rough dose estimations for extracranial tumor lesions are summarized in Table 1.

\section{Posttreatment Observations}

The mean and SD of the administered mass of ${ }^{131} \mathrm{I}$-BA52 was $45 \pm 10 \mu \mathrm{g}$ (range, 20-60 $\mu \mathrm{g}$ ). The mean administered activity was $3,900 \pm 2,000 \mathrm{MBq}$ (range, $510-6,600 \mathrm{MBq}$ ). There were no adverse or clinically detectable pharmacologic effects in any of the 9 treated subjects. Especially, no significant changes in vital signs were observed within $72 \mathrm{~h}$ after administration of the radiopharmaceutical.

Nine patients were selected for therapy with ${ }^{131} \mathrm{I}-\mathrm{BA5} 2$. Because of the limited efficacy of the direct labeling method, the first 4 patients received treatment doses of $4 \mathrm{GBq}$ or less of ${ }^{131} \mathrm{I}-$ BA52 despite the fact that higher doses would have been tolerated based on pretreatment dosimetry. After introduction of the trimethyltin precursor for radiolabeling, treatment activities of 4.3$6.6 \mathrm{GBq}$ were realized in 5 patients. Of these 5 patients, 3 patients survived more than $2 \mathrm{y}$ after therapy. In contrast, the mean overall survival of the untreated and insufficiently dosed patients was approximately $3 \mathrm{mo}$. Some antitumor effect was also demonstrated on diagnostic follow-up imaging (Fig. 4; Supplemental Fig. 2B).

\section{DISCUSSION}

Metastatic malignant melanoma remains an incurable disease in many patients, and the 5-y survival rate for stage IV melanoma is still 15\%-20\% (9). A few years ago, specific tumor geno- and phenotypes were identified that present higher response rates to various targeted treatments, such as the BRAF mutation V600E to treatment with the tyrosine kinase inhibitor vemurafenib, NYESO-1 expression and immunotreatment with Ipilimumab (BristolMyers Squibb), or c-kit mutation to Imatinib (Novartis). However, some patients are or become refractory to those treatments. So there is still a clinical need for further therapeutic options $(10,11)$.

A relevant percentage of human melanomas (12) produce high amounts of the pigment melanin. Consequently, this opens up the 
TABLE 1

Summary of Dosimetric Gy/GBq Values of ${ }^{131}$ I-BA52 Estimated for Organs and Tumor Lesions

\begin{tabular}{|lc|}
\hline \multicolumn{1}{|c}{ Organ } & Dose $(\mathrm{Gy} / \mathrm{GBq})$ \\
\hline Adrenals & 0.35 \\
\hline Brain & 0.19 \\
\hline Breasts & 0.24 \\
\hline Gallbladder wall & 0.43 \\
\hline Lower large intestine wall & 3.10 \\
\hline Small intestine & 0.39 \\
\hline Stomach wall & 0.30 \\
\hline Upper large intestine wall & 4.64 \\
\hline Heart wall & 0.36 \\
\hline Kidneys & 0.30 \\
\hline Liver & 1.30 \\
\hline Lungs & 3.15 \\
\hline Muscle & 0.25 \\
\hline Ovaries & 0.36 \\
\hline Pancreas & 0.34 \\
\hline Red marrow & 0.20 \\
\hline Osteogenic cells & 0.40 \\
\hline Skin & 0.19 \\
\hline Spleen & 0.59 \\
\hline Testes & 0.19 \\
\hline Thymus & 0.29 \\
\hline Thyroid & 0.23 \\
\hline Urinary bladder wall & 0.66 \\
\hline Uterus & 0.30 \\
\hline Total body & 0.33 \\
\hline Effective dose equivalent & 0.99 \\
\hline Effective dose & 0.92 \\
\hline Eyes & \\
\hline Ciliary body & $5.7-15.4$ \\
\hline Choroid & 2.7 \\
\hline Melana & \\
\hline Mean \pm SD & $1.13-12.2$ \\
\hline Range & \\
\hline
\end{tabular}

possibility of treating metastatic melanoma using radionuclidetagged molecules targeting melanin. The efficiency of this targeting depends on the amount and accessibility of the intracellular melanin, which is contained in the melanosomes. Benzamides have been shown to specifically accumulate in melanotic melanoma, which may be used for diagnostic imaging purposes (12-15). In addition, the high uptake capacity allows their use as transporters for cytotoxic agents $(16,17)$. In contrast to melanin-specific antibodies such as 6D2 and 11B11 $(18,19)$, small lipophilic molecules such as ${ }^{131}$ I-BA52 should be able to reach the intracellular melanin through penetration of the cellular membranes by passive diffusion and attain high accumulation rates by trapping in the melanosomes.

In this study, we evaluated the possible application of ${ }^{131} \mathrm{I}-\mathrm{BA} 52$, a benzamide with excellent melanin-binding properties, for the treatment of patients with metastatic melanoma. The synthesis used for preclinical evaluation of the tracer appeared to be insufficiently scalable to fulfill the demands of a routinely clinical application. Therefore, the successful development of the presented precursorbased labeling procedure presents an important interim result of our work. The application of ${ }^{123}$ I- and ${ }^{131}$ I-BA52 was safe and well tolerated, and pharmacologic or allergic side effects were not observed. This is consistent with reports from Michelot et al. using

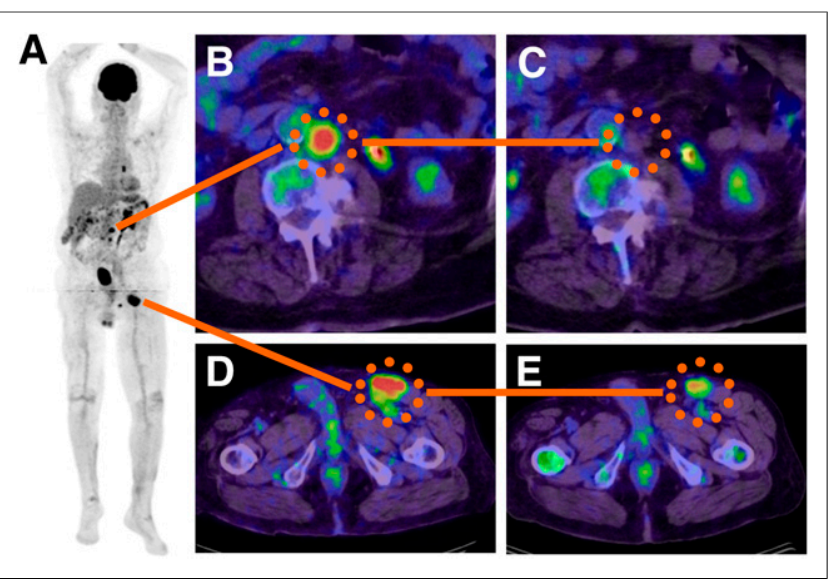

FIGURE 4. A 59-y-old patient with malignant melanoma of left lower extremity causing lymph node metastasis inguinal and paraortal up to mediastinum. ${ }^{18} \mathrm{~F}-\mathrm{FDG}$ PET maximum-intensity projection (A) demonstrates metastases inguinal and paraaortal, both abdominal and mediastinal. One example of paraaortal metastasis (B) presented with intense ${ }^{18} \mathrm{~F}-\mathrm{FDG}$ accumulation (standardized uptake value [SUV], 12.3) in pretherapeutic PET/CT that changed to ${ }^{18} \mathrm{~F}-\mathrm{FDG}$-negative in follow-up PET/CT 6 wk after therapy (C). Inguinal lymph node metastasis with initially intense ${ }^{18} \mathrm{~F}-\mathrm{FDG}$-uptake (D) (SUV, 9.02) decreased in size and metabolism (SUV, 5.81) in posttherapy ${ }^{18} \mathrm{~F}-\mathrm{FDG}$ PET/CT (E).

the derivate ${ }^{125} \mathrm{I}-\mathrm{N}$-(2-diethylaminoethyl) 4-iodobenzamide for imaging in 110 melanoma patients $(12,15)$.

As the amount and accessibility of the target melanin for a particular patient are difficult to predict, patients suited for radionuclide therapy with ${ }^{131}$ I-BA52 were selected using the same molecule labeled with the diagnostic radionuclide ${ }^{123} \mathrm{I}$. Because the quantitative assessment of small tumor lesions is limited by the partial-volume effect and the resolution of the imaging system, a patient was considered BA52-positive if all lesions of sufficient size demonstrated enhanced tumor uptake in the visual inspection of the 24-h-after-injection scans. With this criterion, we found that $38 \%(10 / 26)$ of our patients had tumor lesions targeted by BA52. In comparison, approximately $50 \%$ of melanomas present a BRAF mutation, $35 \%$ a NY-ESO- 1 expression, and $2 \%$ a kit mutation (10, 20,21). Therefore, melanin-targeted agents such as ${ }^{131}$ I-BA52 could be of considerable epidemiologic significance in the field of melanoma therapy.

We already discussed that only tumor lesions with sufficient production of melanins show a relevant uptake of by BA52. One challenge is that the pigmentation of skin lesions often differs from distant metastases (22). Therefore, it is reasonable that only 10 of 26 patients demonstrated targeting, despite the fact that most primary melanomas are melanotic. Our study includes only patients with unresectable stage IV disease-that is, with organ metastases that have not been histologically assessed. However, ${ }^{123}$ I-BA52 scans can be used to stratify therapy only for patients whose organ metastases express the target. Imaging is a favorable way to select patients for therapy because it is noninvasive and avoids the question whether a limited number of biopsy samples are representative for most of a patient's tumor volume.

Within the limitations of the initial dosimetry data, based on conventional planar scintigraphic scans, we originally demonstrated that with ${ }^{131}$ I-BA52 systemic tumor doses as suggested for percutaneous radiation of melanoma (40 Gy) are achievable in some patients while staying within the radiation tolerance of the organs under risk. Assuming tolerance doses of 16-20 Gy to the 
lungs and $1 \mathrm{~Gy}$ to the bone marrow, our data indicate a standardized dose of $5 \mathrm{GBq}$ of ${ }^{131} \mathrm{I}-\mathrm{BA} 52$ to be safe. In our patients, this would translate into mean tumor doses of $27.5 \mathrm{~Gy}$ (range, 7-61 Gy), and on the basis of individual dosimetry higher treatment activities and therefore higher tumor doses are achievable for individual patients.

It cannot be finally concluded whether patients with brain metastases are good candidates for therapy with radionuclidetagged benzamide. Cerebral lesions demonstrated a delayed uptake dynamics, and therefore sufficient dosimetry could not be obtained with the diagnostic surrogate tracer ${ }^{123}$ I-BA52. However, the observed accumulation and therefore potential treatment efficacy of BA52 in brain metastases are attractive topics for further investigation. Right now, there is no evidence whether the uptake in brain metastases can be attributed to a capability of BA52 for crossing the blood-brain barrier or can merely be observed in cases of a blood-brain barrier breakdown at the metastases.

Because of the short physical half-life of the imaging nuclide ${ }^{123}$ I, our tumor dose estimations have to be taken with caution because a relevant error magnification can be introduced by the extrapolation from ${ }^{123} \mathrm{I}$ to ${ }^{131} \mathrm{I}$. Nevertheless, a reliable tumor dose calculation is also provided for a patient under ${ }^{131}$ I-BA52 treatment who received serial imaging up to $120 \mathrm{~h}$ after application and confirmed the favorable tumor-to-nontumor dose distribution. Nevertheless, the high-energy $\gamma$ emission $(364 \mathrm{keV})$ of ${ }^{131}$ I requires imaging with high-energy collimators that reduce imaging resolution and vice versa constrains the quantitative evaluation of small metastases by increasing the partial-volume effect or qualitative assessment whether the tumor uptake within 1 or between 2 particular lesions is rather homogeneous or heterogeneous. However, BA52 can also be labeled with the PET isotope ${ }^{124} \mathrm{I}$, which provides both a sufficient long half-life $(100 \mathrm{~h})$ for dosimetry and improved spatial resolution due to the superior imaging modality. Currently, the availability of ${ }^{124} \mathrm{I}$ increases, and hopefully PET-based imaging will provide a more reliable basis for dosimetry and tumor uptake assessment in the future investigation of radioiodinated benzamides.

Despite the fact that the surrogate treatment of end-stage patients without further established options presents the earliest phase of human investigation, we already observed (radiologic measurement) measurable antitumor effects and a surprisingly long survival of more than $2 \mathrm{y}$ in 3 of 5 patients who received sufficient amounts of the radiopharmaceutical. Such observations are not common in phase 0/1 trials and support radioiodinated BA52-benzamide as a promising agent that merits further investigation.

\section{CONCLUSION}

Our data indicate systemic radionuclide therapy with ${ }^{131}$ I-BA52 as a novel approach for the treatment of malignant melanoma is of considerable potential. The dosimetry of ${ }^{131}$ I-BA52 and moderate side effects underline the specificity of this treatment. Future trials should be done to enhance the precision of dosimetry, validate a maximum tolerable dose, and evaluate the effectiveness of the treatment in a prospective manner.

\section{DISCLOSURE}

The costs of publication of this article were defrayed in part by the payment of page charges. Therefore, and solely to indicate this fact, this article is hereby marked "advertisement" in accordance with 18 USC section 1734 . No potential conflict of interest relevant to this article was reported.

\section{ACKNOWLEDGMENTS}

We are grateful to Heribert Hänscheid and Michael Lassmann, University of Würzburg, Germany, for their support in the dosimetric calculations and to Andreas Nagel, University Hospital Heidelberg, Germany, for the calculation of the effective half-lives. We thank William E. Hull, German Cancer Research Center Heidelberg, Germany, for the comprehensive NMR analysis of the precursors. Furthermore, we thank the Nuclear Medicine Technicians for their effort in the collection of the clinical date and the patient care.

\section{REFERENCES}

1. Jemal A, Devesa SS, Hartge P, Tucker MA. Recent trends in cutaneous melanoma incidence among whites in the United States. J Natl Cancer Inst. 2001;93:678-683.

2. Jemal A, Siegel R, Ward E, et al. Cancer statistics, 2006. CA Cancer J Clin. 2006;56:106-130.

3. Markovic SN, Erickson LA, Rao RD, et al. Malignant melanoma in the 21st century, part 2: staging, prognosis, and treatment. Mayo Clin Proc. 2007;82:490-513.

4. Sun W, Schuchter LM. Metastatic melanoma. Curr Treat Options Oncol. 2001;2:193-202.

5. Croghan GA, Suman VJ, Maples WJ, et al. A study of paclitaxel, carboplatin, and bortezomib in the treatment of metastatic malignant melanoma: a phase 2 consortium study. Cancer. 2010;116:3463-3468.

6. Schild SE. Role of radiation therapy in the treatment of melanoma. Expert Rev Anticancer Ther. 2009;9:583-586.

7. Eisenhut M, Mohammed A, Mier W, et al. Melanoma uptake of ${ }^{99 \mathrm{~m}} \mathrm{Tc}$ complexes containing the $\mathrm{N}$-(2-diethylaminoethyl)benzamide structural element. $\mathrm{J}$ Med Chem. 2002;45:5802-5805.

8. Stabin MG, Sparks RB, Crowe E. OLINDA/EXM: the second-generation personal computer software for internal dose assessment in nuclear medicine. $\mathrm{J} \mathrm{Nucl}$ Med. 2005;46:1023-1027.

9. Recchia F, Candeloro G, Necozione S, Fumagalli L, Bratta M, Rea S. Multicenter phase II study of chemoimmunotherapy in the treatment of metastatic melanoma. Anticancer Drugs. 2008;19:201-207.

10. Chapman PB, Hauschild A, Robert C, et al. Improved survival with vemurafenib in melanoma with BRAF V600E mutation. N Engl J Med. 2011;364:2507-2516.

11. Robert C, Thomas L, Bondarenko I, et al. Ipilimumab plus dacarbazine for previously untreated metastatic melanoma. N Engl J Med. 2011;364:2517-2526.

12. Michelot JM, Moreau MF, Veyre AJ, et al. Phase II scintigraphic clinical trial of malignant melanoma and metastases with iodine-123-N-(2-diethylaminoethyl 4-iodobenzamide). J Nucl Med. 1993;34:1260-1266.

13. Larisch R, Schulte KW, Vosberg H, Ruzicka T, Muller-Gartner HW. Differential accumulation of iodine-123-iodobenzamide in melanotic and amelanotic melanoma metastases in vivo. $\mathrm{J} \mathrm{Nucl} \mathrm{Med.} \mathrm{1998;39:996-1001.}$

14. Denoyer D, Potdevin T, Roselt $P$, et al. Improved detection of regional melanoma metastasis using ${ }^{18} \mathrm{~F}-6$-fluoro-N-[2-(diethylamino)ethyl] pyridine-3-carboxamide, a melanin-specific PET probe, by perilesional administration. $J$ Nucl Med. 2011;52:115-122.

15. Michelot JM, Moreau MF, Labarre PG, et al. Synthesis and evaluation of new iodine-125 radiopharmaceuticals as potential tracers for malignant melanoma. $J$ Nucl Med. 1991;32:1573-1580.

16. Oltmanns D, Eisenhut M, Mier W, Haberkorn U. Benzamides as melanotropic carriers for radioisotopes, metals, cytotoxic agents and as enzyme inhibitors. Curr Med Chem. 2009;16:2086-2094.

17. Wolf M, Bauder-Wust U, Mohammed A, et al. Alkylating benzamides with melanoma cytotoxicity. Melanoma Res. 2004;14:353-360.

18. Dadachova E, Nosanchuk JD, Shi L, et al. Dead cells in melanoma tumors provide abundant antigen for targeted delivery of ionizing radiation by a mAb to melanin. Proc Natl Acad Sci USA. 2004;101:14865-14870.

19. Revskaya E, Jongco AM, Sellers RS, et al. Radioimmunotherapy of experimental human metastatic melanoma with melanin-binding antibodies and in combination with dacarbazine. Clin Cancer Res. 2009;15:2373-2379.

20. Velazquez EF, Jungbluth AA, Yancovitz M, et al. Expression of the cancer/testis antigen NY-ESO-1 in primary and metastatic malignant melanoma (MM): correlation with prognostic factors. Cancer Immun. 2007;7:11-17.

21. Willmore-Payne C, Holden JA, Tripp S, Layfield LJ. Human malignant melanoma: detection of BRAF- and c-kit-activating mutations by high-resolution amplicon melting analysis. Hum Pathol. 2005;36:486-493.

22. Schiffner S, Chen S, Becker JC, Bosserhoff AK. Highly pigmented $\operatorname{Tg}(\mathrm{Grm} 1)$ mouse melanoma develops non-pigmented melanoma cells in distant metastases. Exp Dermatol. 2012;21:786-788. 